Bull. Mater. Sci., Vol. 29, No. 6, November 2006, pp. 581-586. (C) Indian Academy of Sciences.

\title{
Towards a versatile platform based on magnetic nanoparticles for in vivo applications
}

\author{
E DUguet*, S VASSEur, S MORNet, g Goglio, A DeMOURgues, J PORTIER, \\ F GRASSET ${ }^{\dagger}, P$ VEVERKA ${ }^{\dagger \dagger}$ and E POLLERT ${ }^{\dagger \dagger}$ \\ Institut de Chimie de la Matière Condensée de Bordeaux, UPR CNRS 9048, Université Bordeaux-1, 87 ave du \\ Dr Albert Schweitzer, 33608 Pessac Cedex, France \\ ${ }^{\dagger}$ Verres et Céramiques, UMR CNRS 6512, Université de Rennes-1, Institut de Chimie de Rennes, CS 74205 , \\ 35042 Rennes Cedex, France \\ ${ }^{\dagger \dagger}$ Institute of Physics, ASCR, 16253 Praha 6, Czech Republic
}

\begin{abstract}
Magnetic nanoparticles have attracted wide attention because of their usefulness as contrast agents for magnetic resonance imaging (MRI) or colloidal mediators for cancer magnetic hyperthermia. This paper examines these in vivo applications through an understanding of the problems involved and the current and future possibilities for resolving them. A special emphasis is made on magnetic nanoparticle requirements from a physical viewpoint, the factors affecting their biodistribution and the solutions envisaged for enhancing their half-life in the blood compartment and targeting tumour cells. Then, our synthesis strategies are presented and focused on covalent platforms based on maghemite and dextran and capable to be tailorderivatized by surface molecular chemistry. The opportunity of taking advantage of temperature-dependence of magnetic properties of some complex oxides for controlling the in vivo temperature is also discussed.
\end{abstract}

Keywords. Magnetic nanoparticle; surface functionalization; in vivo biodistribution; magnetic resonance imaging (MRI); magnetic hyperthermia; contrast agent.

\section{Introduction}

The first medical uses of magnetite powder for internal applications were reported in the 10th century A.D. (Häfeli 1998). During the last decade, miniaturization of electromagnets, development of superconducting electromagnets and introduction of strong permanent magnets ( $\mathrm{Sm}-\mathrm{Co}$ and $\mathrm{Nd}-\mathrm{Fe}-\mathrm{B}$ ) have stimulated the medical use of magnets in fields as diverse as dentistry, cardiology, neurosurgery, oncology, radiology, etc.

In particular, medical uses of magnets extend to modern diagnostic methods such as magnetic resonance imaging (MRI), which is routinely used for three-dimensional noninvasive scans of the human body and is currently the most important diagnostic method available. Early in the development of MRI, it was thought that contrast agents would not be necessary, but it has become increasingly clear that, in many clinical situations, contrast agents can greatly improve the diagnostic value of MRI. Indeed, one of the most effective techniques for altering the relaxivity of water is to introduce a high spin paramagnetic metal complex, e.g. intravenously injected $\mathrm{Gd}^{3+}$-chelates are routinely used as MR contrast agents. More recently, aqueous dispersions of magnetite nanoparticles embedded in dextran corona have been designed for a similar task (Weissleder et al 1990).

*Author for correspondence (duguet@icmcb-bordeaux.cnrs.fr)
Furthermore, the therapeutic potential of magnetism has arisen when hyperthermia, i.e. heat treatment, has been recognized as a promising form of cancer therapy, particularly in synergy with chemo- and/or radiotherapy. As the healing power of heat has been established for a very long time and used to cure a variety of different diseases (Andrä 1998), a novel hyperthermia route for homogeneously treating deep or scattered tumours would consist in concentrating magnetic nanoparticles around and inside the tumorous tissue and making them heat through energy absorption from an external a.c. magnetic field (magnetic fluid hyperthermia, MFH).

Recent reviews were dedicated to the description of these in vivo applications of magnetic nanoparticles including the understanding of the problems involved from the viewpoint of their overall requirements, for their synthesis and bulk and surface properties (Bahadur and Giri 2003; Berry and Curtis 2003; Pankhurst et al 2003; Tartaj et al 2003; Weinmann et al 2003; Mornet et al 2004a; Gupta and Gupta 2005; Sonvico et al 2005a). The aim of this paper is to summarize comprehensively these requirements and present our own synthesis strategies.

\section{MRI physical background}

MRI is based on NMR signal of hydrogen atoms present in the body tissues through the combined effect of a strong static magnetic field, $B_{0}$, up to $2 T$ in current clinical ap- 
paratus and a transverse radiofrequency-field (rf-field) (5-100 MHz) (Pankhurst et al 2003). After the rf-sequence, the net magnetization vector is once again influenced by $B_{0}$ and tries to re-align with it along the longitudinal axis as protons attempt to return to a state of equilibrium. This relaxation phenomenon can be divided into two different independent processes: (i) longitudinal relaxation is the return of longitudinal magnetization in alignment with $B_{0}$ and is termed $T_{1}$-recovery and (ii) transverse relaxation is the vanishing of transverse magnetization and is termed $T_{2}$ decay. Thanks to sequence parameters such as the repetition time and delay time, the operator obtains the desired type of image contrast ( $T_{1}$ - or $T_{2}$-weighted images).

Due to their different $T_{1}$ and $T_{2}$ values, tissues may be differentiated. But, in many clinical situations, these intrinsic differences are small and exogenous contrast media are currently used for a better delineation of tissues. Although these contrast agents may also be administered by inhalation, oral or interstitial routes (Okuhata 1999), only intravenous administration will be discussed in this section. The first generation of these contrast agents consists of $T_{1}$ agents i.e. high spin paramagnetic ions, usually $\mathrm{Gd}^{3+}$, in very stable chelate form obtained through complexation by low molecular weight chelating molecules, such as diethylenetriaminepentaacetic acid. Gd-chelates have no selective extracellular distribution before their excretion by the kidney. Hydrogen atoms of water in proximity to such chelates experience a faster $T_{1}$-relaxation. Consequently, differences in agent concentration result in contrast enhancement on $T_{1}$-weighted images ('positive' contrast). Magnetic nanoparticles, with a size generally between 3 and $10 \mathrm{~nm}$, have also been developed as contrast agents (Bonnemain 1998). The superparamagnetic behaviour of these subdomain magnetic cores is similar to that of paramagnetic substances, in that they lose their magnetization when the magnetic field is removed, but differs by the value of the magnetic moment which is markedly higher. Therefore, their relaxivities are much higher than those of Gd-chelates. In most situations, they are used for their significant capacity to produce predominantly $T_{2}$ relaxation effects, which result in signal reduction on $T_{2}$-weighted images ('negative' contrast). Basically, the phenomenon may be described from the large magnetic field heterogeneity around the nanoparticle through which water molecules diffuse (Okuhata 1999). Diffusion induces dephasing of the proton magnetic moments resulting in $T_{2}$ shortening. Such contrast agents are also called susceptibility agents because of their effect on the magnetic field. $T_{2}$ shortening is a remote effect, whereas $T_{1}$ shortening process requires a close interaction between water molecules and $T_{1}$-agents.

\section{MFH physical background}

Magnetic fluid hyperthermia is based on nanoscale mediators in the form of intravenously injectable colloidal disper- sion of magnetic particles. The origin of magnetic heating via inductive mediators essentially depends on the size and magnetic properties of particles (Andrä 1998).

For multidomain ferro- or ferrimagnetic materials, heating is due to hysteresis losses. Indeed, large particles of such materials contain several sub-domains, each of them having a definite magnetization direction. When exposed to a magnetic field, the domain with magnetization direction along the magnetic field axis grows and the other ones shrink. This phenomenon is called 'domain wall displacements'. As this phenomenon is not reversible, i.e. magnetization curves for increasing and decreasing magnetic field amplitudes do not coincide, the material is said to exhibit 'hysteresis behaviour' and produces heat under a.c. magnetic field.

In sub-domain particles (superparamagnetic particles) no heating due to hysteresis losses can occur because there is no domain wall. In this case, an external a.c. magnetic field supplies energy and assists magnetic moments to rotate in overcoming the energy barrier, $E=K V$, where $K$ is the anisotropy constant and $V$ the volume of the magnetic core. This energy is dissipated when the particle moment relaxes to its equilibrium orientation (Néel relaxation).

For both types of particles, heating can also be due to the rotational Brownian motion within a carrier liquid, i.e. the rotation of the magnetic particle as a whole because of the torque exerted on the magnetic moment by the external a.c. magnetic field. In this case, the energy barrier for reorientation of a particle is determined by rotational friction within the surrounding liquid.

Whatever the origin of heating, delivered heat must be measured in order to compare efficiency of these mechanisms. Specific absorption rate (SAR), also denoted as specific loss power, is defined as the power of heating of a magnetic material per gram. SAR is measured as

$$
\mathrm{SAR}=\left(C_{\text {solvent }} / x_{\text {magnetic element }}\right) *(\mathrm{~d} T / \mathrm{d} t),
$$

where $C_{\text {solvent }}$ is the specific heat capacity of water $(4 \cdot 18 \mathrm{~J} / \mathrm{g} / \mathrm{K}), x_{\text {magnetic element }}$ the weight fraction of magnetic element and $\mathrm{d} T / \mathrm{d} t$ the initial slope of temperature versus time dependence.

With regard to the electromagnetic devices used for magnetic hyperthermia, the technology of a.c. magnetic field is still under development. For biomedical purpose, the frequency has to be superior to $50 \mathrm{kHz}$ to avoid neuromuscular electrostimulation and lower than $10 \mathrm{MHz}$ for appropriate penetration depth of the rf-field. At least one full sized human prototype has been built by MFH Hyperthermiesystem $\mathrm{GmbH}$ (Berlin) and recently used for first clinical trials (Gneveckow et al 2004).

\section{Physiological background}

Intravenous administration is the most useful method to reach any target organ or tissue, because all vital cells 
receive supplies by means of the blood circulation. So, whatever the applications in both MRI and MFH, the use of magnetic nanoparticles in the blood compartment depends also on specific requirements with respect to their plasma half-life and their final biodistribution. The problem of the non-natural stealthiness of nanoparticles towards the immune system and the possibilities for resolving it have been widely studied in the field of drug delivery from polymeric nanoparticles and liposomes (Moghimi and Szebeni 2003). In a very rough way, the immune system may be described from the mononuclear phagocyte system (MPS), i.e. the cell family comprising bone marrow progenitors, blood monocytes and tissue macrophages. These macrophages are widely distributed and strategically placed in many tissues of the body to recognize and clear senescent cells, invading micro organisms or particles. The recognition/clearance mechanism is based on (i) the spontaneous adsorption of circulating plasma proteins (opsonins) capable of interacting with the specialized plasma membrane receptors on monocytes and macrophages, (ii) the particle recognition and (iii) the endocytosis/phagocytosis by these cells, leading to their elimination from circulation and their simultaneous concentration in organs with high phagocytic activity (figure 1). Therefore, after intravenous administration, colloidal particles are cleared within minutes from the bloodstream and their typical final biodistribution is $80-90 \%$ in liver, $5-8 \%$ in spleen and $1-2 \%$ in bone marrow (Monfardini and Veroneses 1998). It provides an opportunity for the efficient delivery of therapeutic agents to these phagocytic cells and therefore, to the related organs. Such an MPS-mediated targeting is called 'passive targeting'.

Nevertheless, if monocytes and macrophages in, or in contact with, blood are not the desired target, a strategy of 'active targeting' has to be developed and the inescapable requirements consist in (i) minimizing or delaying the nanoparticle uptake by MPS and (ii) increasing the probability of redirecting long-circulating particles to the desired target by surface labelling with specific ligands.

Long-circulating nanoparticles are necessarily macrophage-evading nanoparticles and one of the most efficient strategies to design stealthy particles consists in preventing

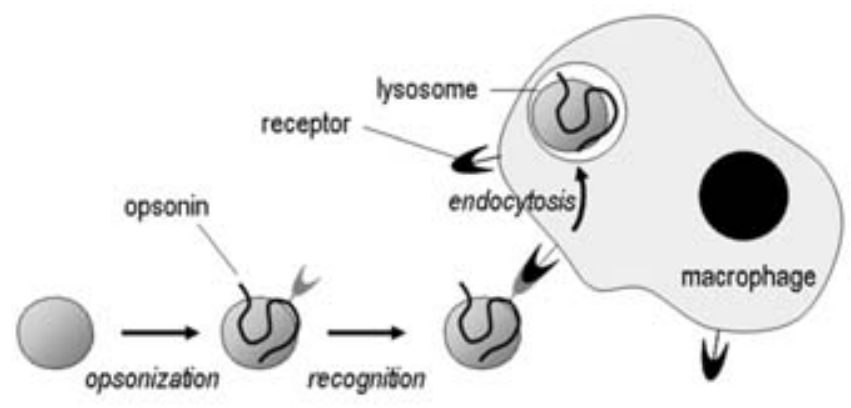

Figure 1. Schematic displaying the main steps of the MPSmediated clearance of non-stealthy particles. opsonin adsorption. It is now well admitted that the smaller the particle and the more neutral and hydrophilic its surface, the longer is its plasma half-life. The hydrophilicity is generally provided by surface corona made of hydrophilic macromolecules for creating polymer brushes, acting as a steric surface barrier and reducing opsonin adsorption. Among these macromolecules, poly(ethylene glycol) is widely used (PEGylation) (Moghimi et al 2001).

For increasing the probability of redirecting longcirculating particles to the desired target, their surface has to be labelled with ligands that specifically bind to surface epitopes or receptors on the target sites (molecular recognition processes such as antibody-antigen interactions). In the case of cancer hyperthermia therapy, active targeting could allow the selective destruction of cancer cells, even if they have escaped the tumour mass and disseminated as metastatic cells. For MR diagnosis, there is also a great need of labelled contrast agents, which would accumulate highly and specifically in malignant tumours, allowing an accurate diagnosis at a stage when the disease would be still treatable. Such a strategy is called 'molecular imaging'. The antibody (or antibody fragments) coupling has at least two drawbacks: the overall dimensions of the antibodies ( $20 \mathrm{~nm})$, which cause particles to diffuse poorly through biological barriers, and their immunogenicity, i.e. the property of being able to evoke an immune response within an organism. For this reason the coupling of small number of immunogenic ligands has also been investigated: oligosaccharides, oligopeptides, folic acid, etc.

\section{Magnetic nanoparticles currently used for MRI or MFH applications}

MRI colloidal $T_{2}$-agents are often called (U)SPIO for (ultrasmall) super paramagnetic iron oxide. They consist of iron oxide cores, whose composition and physicochemical properties vary continuously from magnetite $\mathrm{Fe}_{3} \mathrm{O}_{4}$ to maghemite $\gamma-\mathrm{Fe}_{2} \mathrm{O}_{3}$. For intravenous administration, they are generally synthesized in a one-step process by alkaline coprecipitation of iron (II) and iron (III) precursors in aqueous solutions of hydrophilic macromolecules, e.g. dextran (Molday 1984). These macromolecules serve (i) to limit the magnetic core growth during the synthesis, (ii) to stabilize via sterical repulsions the nanoparticle dispersion in water (and later in physiological medium) and (iii) to reduce the in vivo opsonization process. These colloidal contrast agents would be more realistically described as several magnetic cores, more or less aggregated, embedded in the hydrophilic macromolecules. The overall hydrodynamic diameter is largely higher than the magnetic core dimensions and the size polydispersity is narrowed by fractionation steps.

Two different classes of iron oxides are currently clinically approved or in phase-III trials. SPIO agents exhibit a high $T_{1} / T_{2}$ relaxivity ratio and, because of their overall 
size (over $40 \mathrm{~nm}$ in diameter), they are efficiently accumulated in MPS-organs $(\sim 80 \%$ of the injected dose in liver and $5-10 \%$ in the spleen with plasma half-life lower than $10 \mathrm{~min}$ ). Therefore, SPIO decreases liver and spleen signal within several minutes after intravenous administration. Malignant tumours or metastases, which are typically devoid of a substantial number of Kupffer cells (liver macrophages), appear as hyperintense lesions contrasted against the hypointense liver on $T_{2}$-weighted sequences (Wallis and Gilbert 1999).

USPIO, also called MION for monocristalline iron oxide nanocompound, exhibits an overall hydrodynamic diameter of $<40 \mathrm{~nm}$. Thanks to their smaller size, they act as stealth particles. Their plasma half-life is higher than $2 \mathrm{~h}$ (Bonnemain 1998) and therefore, they remain in the blood long enough to act as blood-pool agents for MR angiography (MRA). Some particles leak to the interstitium, where they are cleared by the macrophages of the lymphatic system or drained via the lymphatic system and subsequently accumulated in the lymph nodes. Therefore, they allow diagnosing hyperplastic and tumorous lymph node by MR lymphography. In comparison with SPIO, USPIO exhibits lower relaxivities but the lower $T_{1} / T_{2}$ ratio leads to a higher contrast on $T_{2}$-weighted images. This $T_{1} / T_{2}$ ratio is also much more favourable for MRA or for low-field $T_{1^{-}}$ weighted MR techniques $\left(B_{0}\right.$ lower than $\left.0 \cdot 5 \mathrm{~T}\right)$.

Interactions between magnetic cores and macromolecules are weak (essentially Van der Waals and hydrogen interactions) (Jung 1995) and generally prevent any efficient derivatization of dextran corona without macromolecule depletion (Groman and Josephson 1993). Dextran crosslinking in a second step appeared to be an efficient way for enhancing the mechanical entrapment of magnetic particles (CLIO for cross-linked iron oxide) (Hogemann et al 2000). Ligand-mediated MR contrast agents were designed in particular for tumour diagnosis (Mornet et al 2004a). Investigations in small animals revealed that it is possible to achieve a high concentration of the magnetic label at the target. However, the required dose of the labelled antibody is still too high to make a commercial development realistic (Weinmann et al 2003). Folatemediation also appeared promising for tumour MRI diagnostics, because folic acid is a vitamin B essential for cell division processes and therefore, folate receptors are frequently overexpressed onto the surface of human cancer cells (Zhang et al 2002).

Since the pioneering work of Gilchrist et al in 1957, magnetic hyperthermia has been the aim of numerous in vitro and in vivo investigations (Moroz et al 2002; Mornet et al 2004a). Currently, the most advanced strategy is developed by Jordan and colleagues in Berlin. Magnetite nanoparticles are modified with aminosilane groups leading to largely positive surface charges in physiological conditions and directly injected in the tumours, restricting this strategy to solid ones (Jordan et al 1999). Hyperthermia sequences $(\sim 100 \mathrm{kHz}-0 \cdot 18 \mathrm{kA} / \mathrm{m})$ are alternated with conventional radiotherapy ones which led to hopeful results in a first series of clinical experiences (Johannsen et al 2003). Further developments will consist to move to active targeting strategy for treating so efficiently small and scattered tumours.

\section{Our method for synthesis and functionalization of ultrasmall superparamagnetic covalent carriers based on maghemite and dextran}

A new generation of $T_{2}$-agents, based on maghemite cores covalently bonded to dextran macromolecules, was recently investigated in our group and called VUSPIO for versatile USPIO (Mornet et al 2004b, 2005). Their preparation consists first the colloidal maghemite synthesis, its surface modification through the grafting of aminopropylsilane groups, $(-\mathrm{O})_{3} \mathrm{Si}-\mathrm{CH}_{2}-\mathrm{CH}_{2}-\mathrm{CH}_{2}-\mathrm{NH}_{2}$ and the coupling of partially oxidized dextran via formation of a Schiff's base bond which is subsequently reduced (figure 2a). Such a step-by-step synthesis permits to control (i) the magnetic core size from $2-10 \mathrm{~nm}$, (ii) the size distribution and (iii) the overall hydrodynamic diameter (figure 2b), thanks to accurate and reproducible experimental conditions, e.g. colloidal stability control, dextran molecular weight and concentration, etc. No fractionation was needed for narrowing the size polydispersity. The presence of $\mathrm{Fe}-\mathrm{O}-\mathrm{Si}$ bonds remains hypothetical (no evidence through IR spectroscopy), but the covalent anchoring of aminopropylsilane groups is mainly due to their self polycondensation leading to a highly cross-linked polysiloxane film entrapping each maghemite nanoparticle.

Extensive study of VUSPIO relaxivities as a function of the magnetic core size and concentration is currently in progress. Preliminary results displayed MR behaviour very similar to that of conventional USPIO.

These stable agents may be easily derivatized for surface labelling, e.g. PEGylation, ligand coupling, etc. For instance, folate-labelled VUSPIO were designed for tumour MR diagnosis or MFH treatments (figure 3). A controlled amount of acid folic-conjugated PEG macromolecules was chemically bound to the dextran corona of VUSPIO whose surface was subsequently saturated using grafted PEG in order to reduce the opsonization process. A final overall hydrodynamic diameter of $\sim 50 \mathrm{~nm}$ was found. Cell culture contact was performed with three cell lines previously defined for their expression of the specific folate receptor. Folate-labelled VUSPIO were internalized only in the folate receptor-bearing cells and PEGylated but unlabelled VUSPIO were not internalized, whatever the cell lines (Sonvico et al 2005b). Magnetic heating experiments $(108 \mathrm{kHz}-69 \mathrm{kA} / \mathrm{m})$ were performed in folate-labelled VUSPIO concentration conditions similar to those found in cells $(3 \mathrm{mg} / \mathrm{mL})$ and showed a heating ramp of $7 \pm$ $1{ }^{\circ} \mathrm{C} / 20$ min period. The specific absorption rate, SAR, was found equal to $13 \pm 3 \mathrm{~W} / \mathrm{g}_{\mathrm{Fe}}$. These results are in good agreement with those obtained for bare maghemite cores $\left(\mathrm{SAR}=13.8 \mathrm{~W} / \mathrm{g}_{\mathrm{Fe}}\right)$. 


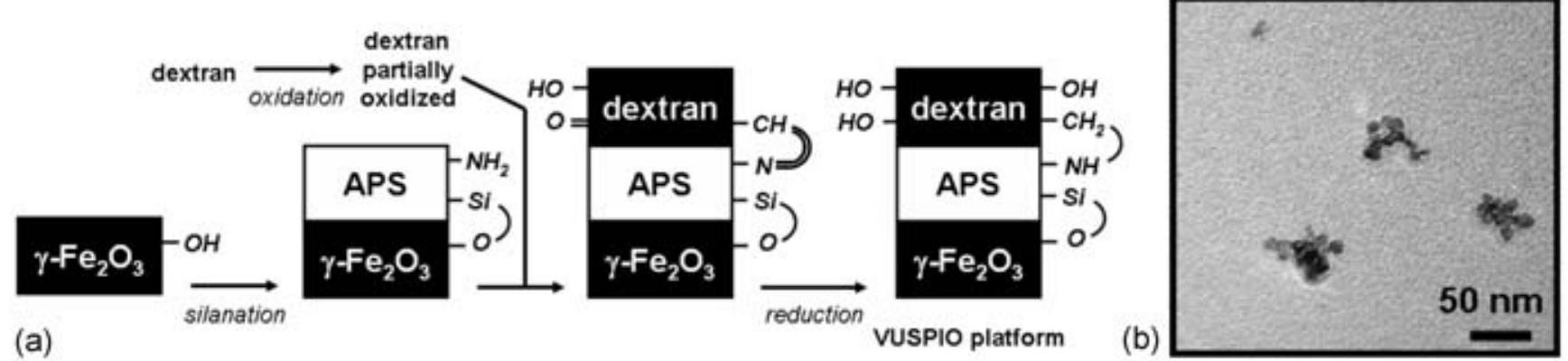

Figure 2. (a) Multistep synthesis route for building VUSPIO platform and (b) transmission electron micrography of negatively stained VUSPIO nanoparticles.

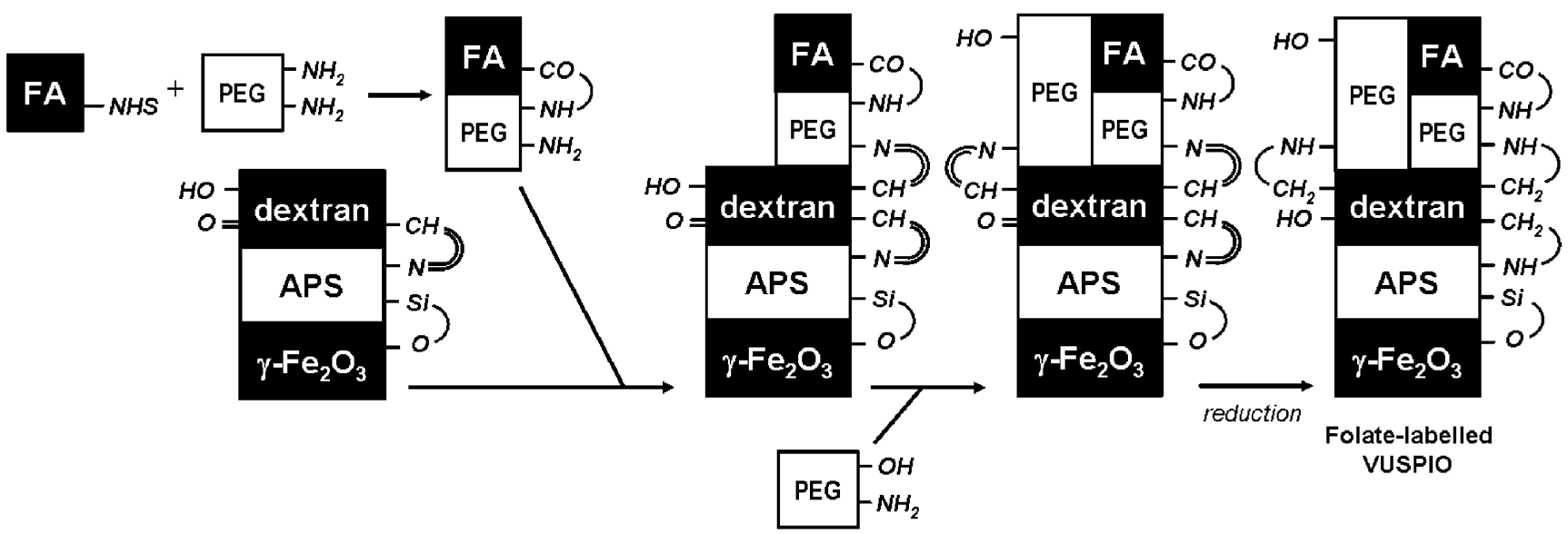

Figure 3. Multistep synthesis route for the labelling of VUSPIO platform by folic acid (note: folic acid was used under the form, $\mathrm{N}$-hydroxysuccinimide ester derivative).

\section{Towards self-controlled heating mediators for magnetic hyperthermia}

One of the last crucial steps for clinical application of magnetic hyperthermia remains the temperature control because on the one hand heat conduction and energy adsorption in vivo are widely unknown and on the other hand local overheating may damage safe tissue.

A promising route could exploit the temperature dependence of magnetic properties. Indeed, Curie temperature $\left(T_{\mathrm{C}}\right)$ is the temperature at which ferromagnetic particles lose their magnetic properties, thus they do not convert electromagnetic energy into heat. Curie temperature is, therefore, the maximal temperature reachable by magnetic particles. Choosing an appropriate Curie temperature would be the smartest way to control hyperthermia because in that case particles would be both heaters and fuses. Such a strategy has already been developed for alloy thermoseeds in order to prevent local tissue overheating and reduce the need for invasive thermometry (Moroz et al 2002).

First, we investigated the potential use of yttrium aluminium iron garnet, $\mathrm{Y}_{3} \mathrm{Fe}_{5-x} \mathrm{Al}_{x} \mathrm{O}_{12}$ nanoparticles, which were synthesized by the citrate gel process by varying the aluminium content, $x$, from 0 to 2 (Grasset et al 2001). The average diameter was $\sim 100 \mathrm{~nm}$ and the Curie temperature range from $-40^{\circ} \mathrm{C}$ (for $x=2$ ) to $280^{\circ} \mathrm{C}$ (for $x=0$ ). Therefore, it is possible to adjust $T_{\mathrm{C}}$ at the temperature necessary for hyperthermia experiments. By interpolation, it was found that it corresponds to a value for $x=1 \cdot 5$. Nevertheless, at body temperature, the magnetization of this compound (which decreases with increasing $x$ value) is probably not high enough for efficient heating.

More recently, manganese perovskite, $\mathrm{La}_{0 \cdot 75} \mathrm{Sr}_{0 \cdot 25} \mathrm{MnO}_{3}$ nanoparticles, of the crystallite size $20-180 \mathrm{~nm}$ were prepared starting from citrate gel precursor by annealing in the range of $570-900^{\circ} \mathrm{C}$ (Vasseur et al 2006). It was shown that the decrease in crystallite size leads to a gradual decrease in the magnetization and Curie temperature. The observed behaviour is a consequence of a non-collinear spin arrangement at the surface layer of crystallites, estimated to be of $\sim 5 \mathrm{~nm}$. Magnetic heating experiments $(108 \mathrm{kHz}-69 \mathrm{kA} / \mathrm{m})$ were carried out with an aqueous stable suspension of a selected sample $\left(M_{1 T}=42 \mathrm{emu} / \mathrm{g}, T_{\mathrm{C}}=352 \mathrm{~K}\right)$. The maximal temperature reached by $\mathrm{La}_{0.75} \mathrm{Sr}_{0.25} \mathrm{MnO}_{3}$ particles was $57.5^{\circ} \mathrm{C}$ (figure 4 ). The yield values of SAR were found to be larger than $500 \mathrm{~W} / \mathrm{g}_{\mathrm{Mn}}$ at $27^{\circ} \mathrm{C}$ and $350 \mathrm{~W} / \mathrm{g}_{\mathrm{Mn}}$ at $37^{\circ} \mathrm{C}$. 


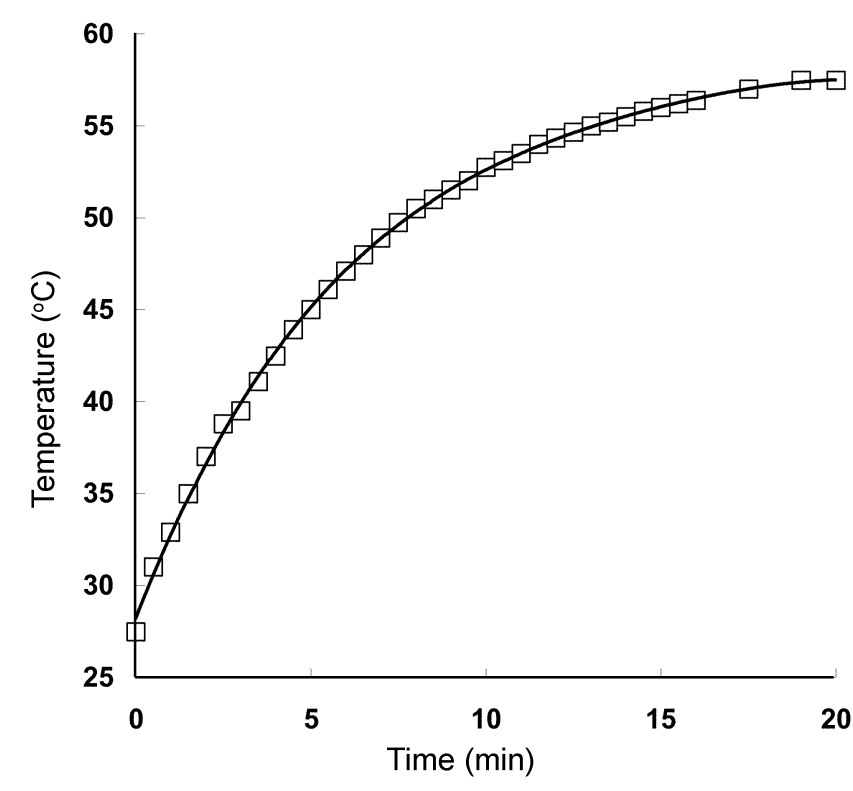

Figure 4. Time course of the temperature inside the measuring cell during magnetic heating using $\mathrm{La}_{0.75} \mathrm{Sr}_{0.25} \mathrm{MnO}_{3}$ nanoparticles as mediators $(108 \mathrm{kHz}-69 \mathrm{kA} / \mathrm{m}-4 \cdot 2 \mathrm{~g} / \mathrm{L})$.

\section{Conclusions}

Magnetic nanoparticles are now routinely used as contrast agents for the MPS-organs (liver, spleen and bone marrow). It is obvious that future developments will be in the direction of active targeting through molecular imaging. Therefore, in the case of cancer diagnosis, the next challenge for the future is the generation of functionalized surfaces of these particles.

At the same time, extensive efforts have led to clinical trials of magnetic hyperthermia by using colloidal mediators. In this field, both challenges will be the design of stealth nanoparticles able to circulate in the blood compartment for a long time and the surface grafting of ligands able to facilitate their specific internalization in tumour cells.

\section{References}

Andrä W 1998 Magnetism in medicine (eds) W Andrä and H Nowak (Weinheim: Wiley-VCH) p. 455
Bahadur D and Giri J 2003 Sadhana 28639

Berry C C and Curtis A S G 2003 J. Phys. D: Appl. Phys. 36 R198 Bonnemain B 1998 J. Drug Targeting 6167

Gneveckow U et al 2004 Med. Phys. 311444 (For more data: http://www.mag-force.de)

Grasset F, Mornet S, Demourgues A, Portier J, Bonnet J, Vekris A and Duguet E 2001 J. Magn. Magn. Mater. 234409

Groman E V and Josephson L 1993 US Patent 5,248,492

Gupta A K and Gupta M 2005 Biomaterials 263995

Häfeli U 1998 Magnetism in medicine (eds) W Andrä and H Nowak (Weinheim: Wiley-VCH) p. 15

Hogemann D, Josephson L, Weissleder R and Basilion J P 2000 Bioconjugate Chem. 11941

Johannsen M et al 2003 Int. J. Hyperthermia 21637

Jordan A, Scholz R, Wust P, Schirra H, Schiestel T, Schmidt H and Felix R 1999 J. Magn. Magn. Mater. 194185

Jung C W 1995 Magn. Reson. Imaging 13675

Moghimi S M and Szebeni J 2003 Prog. Lipid Res. 42463

Moghimi S M, Hunter A C and Murray J C 2001 Pharmacol. Rev. $\mathbf{5 3} 283$

Molday R S 1984 US Patent 4,452,773

Monfardini C and Veroneses F M 1998 Bioconj. Chem. 9418

Mornet S, Vasseur S, Grasset F and Duguet E 2004a J. Mater. Chem. 142161

Mornet S, Duguet E and Portier J 2004b French Patent 2,855,315

Mornet S, Portier J and Duguet E 2005 J. Magn. Magn. Mater. 293127

Moroz P, Jones S K and Gray B N 2002 Int. J. Hyperthermia 18 267

Okuhata Y 1999 Adv. Drug Delivery Rev. 37121

Pankhurst Q A, Connolly J, Jones S K and Dobson J 2003 J. Phys. D: Appl. Phys. 36 R167

Sonvico F, Dubernet C, Colombo P and Couvreur P 2005a Curr. Pharm. Des. 112091

Sonvico F et al 2005b Bioconj. Chem. 161181

Tartaj P, del Puerto Morales M, Veintemillas-Verdaguer S, González-Carreño T and Serna C J 2003 J. Phys. D: Appl. Phys. 36 R182

Vasseur S et al 2006 J. Magn. Magn. Mater. 302315 (doi:10.1016/j.jmmm.2005.09.026)

Wallis F and Gilbert F J 1999 J. R. Coll. Surg. Edinb. 44117

Weinmann H J, Ebert W, Misselwitz B and Schmitt-Willich H 2003 Eur. J. Radiol. 4633

Weissleder R, Elizondo G, Wittenberg J, Rabito C A, Bengele L and Josephson L 1990 Radiology 175489

Zhang Y, Kohler N and Zhang M 2002 Biomaterials 231553 